\title{
Prioridades de investigación en salud en las tesis universitarias en Puno, Perú
}

\author{
Oscar Mamani-Benito* 1; Glubert Ramos-Vilca ${ }^{2}$; Dawer Rodrigo Ccaso ${ }^{3}$; Neyda Chipana Paye 3; Fernando Quispe Mayta ${ }^{4}$; \\ Laura Ccasa-Valero ${ }^{4}$; Christian R. Mejia ${ }^{5}$
}

\section{RESUMEN}

El objetivo de esta investigación fue determinar cuál es la frecuencia con la que se abordan temas de prioridad nacional en investigación en las tesis universitarias en Puno, Perú. Es un estudio transversal y observacional en 482 tesis aprobadas entre 2016 y 2018 de las facultades de medicina (25,10\%), enfermería (46,47\%), nutrición (18,46\%) y psicología (9,95\%) de universidades de Puno. Encontramos una proporción mayor de trabajos descriptivos (56,84 \%) con diseño no experimental $(90,45 \%)$. Las prioridades que se abordaron fueron salud mental (7,05 \%), recursos humanos $(6,22 \%)$, malnutrición y anemia $(18,87 \%)$, mortalidad materna, perinatal y neonatal $(4,77 \%)$, tuberculosis $(1,86 \%)$, cáncer $(5,80 \%)$, hipertensión arterial, dislipidemias y enfermedades cardiovasculares $(3,52 \%)$, políticas y gestión en salud $(0,41 \%)$, diabetes mellitus $(7,46 \%)$ e infecciones respiratorias y neumonías (5,60 \%). Los accidentes de tránsito no se han considerado en este grupo. Concluimos que más del 50 \% de las tesis de las universidades de Puno abordan una prioridad de investigación en salud.

Palabras clave: Tesis académica; Prioridades en salud; Investigación (Fuente: DeCS BIREME).

\section{Health research priorities in university theses in Puno, Peru}

\section{ABSTRACT}

This research aimed to determine the frequency that national health research priority topics are addressed in university theses in Puno, Peru. This was an observational cross-sectional study conducted in 482 theses approved between 2016 and 2018 in universities' schools of medicine (25.10\%), nursing (46.47\%), nutrition (18.46\%) and psychology (9.95\%) in Puno. A greater proportion of descriptive (56.84 \%) and non-experimental research works $(90.45 \%)$ were found. The priority topics were mental health (7.05\%); human resources (6.22\%); malnutrition and anemia (18.87\%); maternal, perinatal and neonatal mortality $(4.77 \%)$; tuberculosis (1.86 \%); cancer (5.80\%); high blood pressure, dyslipidemia and cardiovascular diseases (3.52 \%); health policy and management $(0.41 \%)$; diabetes mellitus $(7.46 \%)$; and respiratory infections and pneumonia (5.60\%). Traffic accidents were not considered in this group. It is concluded that over $50 \%$ of the theses from the universities in Puno address a health research priority topic.

Keywords: Academic dissertation; Health priorities; Research (Source: MeSH NLM).

\footnotetext{
1 Universidad Peruana Unión. Juliaca, Perú.

2 Universidad Andina Néstor Cáceres Velásquez, Asociación Científica de Estudiantes de Medicina. Juliaca, Perú.

3 Universidad Peruana Unión, Escuela Profesional de Psicología. Juliaca, Perú.

4 Universidad Nacional del Altiplano. Puno, Perú.

5 Universidad Continental. Lima, Perú.

* Autor corresponsal.
} 


\section{INTRODUCCIÓN}

En el Perú se está trabajando para mejorar la calidad universitaria (1). No obstante, la producción científica es todavía motivo de preocupación, sobre todo en el pregrado (2). Varias investigaciones en América Latina revelan que nuestro país aporta una proporción muy baja de nuevos conocimientos ${ }^{(3)}$, y que una de las carreras que más genera evidencias en salud es la de Medicina (4). Por otro lado, otras, como enfermería, nutrición y psicología, presentan algunas limitaciones ${ }^{(5)}$.

En nuestro país, durante los últimos años, se ha observado el incremento de algunos problemas sanitarios, por ello, desde al año 2010, el Instituto Nacional de Salud ha planteado las prioridades nacionales de investigación (6), con el fin de orientar los estudios, contrarrestar los efectos en la población y optimizar los limitados recursos para la investigación ${ }^{(7)}$. La primera lista de prioridades apareció en el periodo 2010-2014 (6), fue actualizada para el periodo $2015-2021^{(8)} ; y$ ahora está vigente la del periodo $2019-2023^{(9)}$.

Es necesario indicar que esta iniciativa involucra también a la investigación formativa en las universidades, por lo que las tesis de pregrado deberían alinearse hacia estas prioridades de investigación para generar conocimiento científico y enfrentar estos problemas. Por esta razón, nuestra investigación tiene el objetivo de determinar cuál es la frecuencia con la que las tesis realizadas en las universidades del departamento de Puno abordan las prioridades nacionales de investigación en salud.

\section{EL ESTUDIO}

Se realizó un estudio transversal y observacional de análisis de documentos ${ }^{(10)}$ entre los meses de setiembre a diciembre del año 2019. Las unidades de análisis fueron 482 tesis de las carreras profesionales de medicina, enfermería, nutrición y psicología que fueron aprobadas en el periodo 2016-2018. Este número es el resultado de una búsqueda de trabajos de grado en salud en los repositorios digitales de cuatro universidades del departamento de Puno: Universidad Nacional del Altiplano (UNA), Universidad Andina Néstor Cáceres Velásquez (UANCV), Universidad Peruana Unión (UPeU, campus Juliaca) y Universidad Privada San Carlos (UPSC).

Las características metodológicas de las tesis (tipo de investigación, diseño, tipo de población y tamaño muestral) se registraron en una ficha de recolección de datos. Para ello, se revisó el resumen y el apartado sobre la metodología. La variable principal (el abordaje de prioridades nacionales de investigación) fue verificada al analizar el título del trabajo y el planteamiento del problema.

Se consideraron las siguientes prioridades nacionales de investigación correspondientes al periodo 2015-2021 ${ }^{(8)}$ :

1. Mortalidad materna, perinatal y neonatal

2. Cáncer

3. Malnutrición y anemia

4. Salud mental

5. Hipertensión arterial, dislipidemias, enfermedades cardiovasculares

6. Políticas y gestión en salud

7. Diabetes mellitus

8. Tuberculosis

9. Recursos humanos

10. Infecciones respiratorias y neumonía

11. Accidentes de tránsito

La base de datos se completó con el programa Microsoft Excel (versión 2016), luego fue verificada y se hizo un control de calidad. Los resultados se presentaron en tablas de frecuencia y porcentaje. Esta investigación no requirió la aprobación de un comité de ética en investigación, pues las tesis registradas en cada repositorio digital son de acceso libre para el público en general; no obstante, se mantuvo en reserva las identidades del autor y del asesor de las tesis.

\section{RESULTADOS}

El mayor número de las tesis fueron aprobadas en el año 2017 (42,53\%) y corresponden a las facultades de Enfermería $(46,47 \%)$, Medicina (25,10\%), Nutrición (18,46\%) y Psicología $(9,95 \%)$. Por otro lado, el $64,52 \%$ pertenecen a egresados de la Universidad Nacional del Altiplano; el 19,29\%, a la Universidad Andina Néstor Cáceres Velásquez; el 15,14\%, a la Universidad Peruana Unión, y 1,03 \%, a la Universidad Privada San Carlos (Tabla 1).

En cuanto a las características metodológicas, el 36,09\% incluyó población hospitalaria y el $23,85 \%$, escolares. Además, el $56,84 \%$ son estudios descriptivos y $28,21 \%$ son de tipo correlacional, lo cual concuerda con el diseño predominante que, en este caso, es no experimental $(90,45 \%)$. Por último, el tamaño de muestra más frecuente se ubica entre 51 a 200 participantes $(58,71 \%)$. 
Prioridades de investigación en salud en las tesis universitarias en Puno, Perú

Tabla 1. Características de las tesis de salud de universidades de Puno (2016-2018)

\begin{tabular}{|c|c|c|c|}
\hline Variable & Categoría & $f$ & $\%$ \\
\hline \multirow[t]{4}{*}{ Carrera profesional } & Enfermería & 224 & 46,47 \\
\hline & Medicina & 121 & 25,10 \\
\hline & Nutrición & 89 & 18,46 \\
\hline & Psicología & 48 & 9,95 \\
\hline \multirow[t]{3}{*}{ Año de aprobación } & 2016 & 81 & 16,80 \\
\hline & 2017 & 205 & 42,53 \\
\hline & 2018 & 196 & 40,66 \\
\hline \multirow[t]{4}{*}{ Universidad } & UNA & 311 & 64,52 \\
\hline & UANCV & 93 & 19,29 \\
\hline & UPeU & 73 & 15,14 \\
\hline & UPSC & 5 & 1,03 \\
\hline \multirow[t]{7}{*}{ Tipo de población } & Escolar & 115 & 23,85 \\
\hline & Universitario & 41 & 8,50 \\
\hline & Centro de salud & 70 & 14,52 \\
\hline & Comunidad & 26 & 5,39 \\
\hline & Hospitalario & 174 & 36,09 \\
\hline & Penitenciario & 6 & 1,24 \\
\hline & Otro & 50 & 10,37 \\
\hline \multirow[t]{4}{*}{ Tipo de investigación } & Descriptivo & 274 & 56,84 \\
\hline & Correlacional & 136 & 28,21 \\
\hline & Explicativo & 18 & 3,73 \\
\hline & Otra & 54 & 11,20 \\
\hline \multirow[t]{2}{*}{ Diseño } & Experimental & 46 & 9,54 \\
\hline & No experimental & 436 & 90,45 \\
\hline Tamaño muestral & Hasta 50 & 130 & 26,97 \\
\hline \multirow[t]{4}{*}{ (participantes) } & Entre 51 a 200 & 283 & 58,71 \\
\hline & Entre 201 a 400 & 60 & 12,44 \\
\hline & Entre 401 a 600 & 3 & 0,62 \\
\hline & Más de 600 & 6 & 1,24 \\
\hline
\end{tabular}

La tabla 2 muestra que 297 tesis $(61,62 \%)$ abordaron alguna prioridad de estudio de salud y que la más frecuente fue malnutrición y anemia (18,87 \%), seguida por diabetes mellitus (7,46\%), salud mental $(7,05 \%)$, recursos humanos $(6,22 \%)$, mortalidad materna, perinatal y neonatal (4,77 \%), cáncer (5,80\%), infecciones respiratorias y neumonía (5,60\%), hipertensión arterial, dislipidemias y enfermedades cardiovasculares (3,52\%), tuberculosis $(1,86 \%)$ y políticas y gestión en salud $(0,41 \%)$, y ninguna tesis incluye accidentes de tránsito.

Tabla 2. Inclusión de las prioridades de investigación en las tesis de salud de universidades de Puno

\begin{tabular}{|lrrr|}
$\begin{array}{c}\text { Prioridades de investigación } \\
\text { en salud: 2016-2021 }\end{array}$ & ilncluye? & f & $\%$ \\
\hline Salud mental & No & 448 & 92,94 \\
\hline Recursos humanos & Sí & 34 & 7,05 \\
& No & 452 & 93,77 \\
\hline & Sí & 30 & 6,22 \\
\hline
\end{tabular}




\begin{tabular}{|lrrr|}
\hline \multicolumn{1}{|c}{$\begin{array}{c}\text { Prioridades de investigación } \\
\text { en salud: } 2016-2021\end{array}$} & ilncluye? & f & \\
\hline Malnutrición y anemia & No & 391 & 81,12 \\
\hline Mortalidad materna, perinatal y neonatal & Sí & 91 & 18,87 \\
\hline Tuberculosis & No & 459 & 95,22 \\
\hline & Sí & 23 & 4,77 \\
\hline Cáncer & No & 473 & 98,13 \\
\hline & Sí & 9 & 1,86 \\
\hline Hipertensión arterial, dislipidemias, & No & 454 & 94,19 \\
\hline enfermedades cardiovasculares & Sí & 28 & 5,80 \\
\hline Políticas y gestión en salud & No & 465 & 96,47 \\
\hline & Sí & 17 & 3,52 \\
\hline Diabetes mellitus & No & 480 & 99,58 \\
\hline Infecciones respiratorias y neumonía & Sí & 2 & 0,41 \\
\hline & No & 446 & 92,53 \\
\hline Accidentes de tránsito & Sí & 36 & 7,46 \\
\hline & No & 455 & 94,39 \\
\hline
\end{tabular}

\section{DISCUSIÓN}

El 61,62 \% de las tesis analizadas abordaron alguna prioridad de estudio de salud, no obstante, un análisis independiente por cada prioridad evidencia una proporción inferior al $10 \%$, excepto para el caso de malnutrición y anemia, que supera el $15 \%$. Este hallazgo corrobora, en parte, la tendencia ya existente, como el estudio de Quispe-Juli, que reporta una baja inclusión de prioridades de investigación en salud en trabajos de pregrado de una universidad de Arequipa (11). De acuerdo a este hecho y a las necesidades y problemas de salud en el departamento de Puno ${ }^{(12-14)}$, nuestros resultados permiten reflexionar sobre los objetivos de la investigación formativa en esta parte del Perú, y que necesariamente deben orientarse a la solución de los problemas de la región.

Por otro lado, la mayoría de las tesis siguen un diseño no experimental de tipo descriptivo o correlacional. Resultados similares han sido reportados por Mamani ${ }^{(15)}$ en un estudio sobre la calidad de las tesis de psicología en una universidad de Juliaca, y también por la investigación de Castro-Maldonado et al. ${ }^{(16)}$ en las tesis de medicina de una universidad de Lima y, recientemente, por Mamani et al. (17), que analizaron las características de tesis de salud de cuatro universidades del departamento de Puno.

Con base en esta información, inferimos que la motivación de los universitarios que inician la elaboración de sus proyectos de tesis es terminar el proceso en el menor tiempo posible y no complicarse con cuestiones metodológicas (18) o procedimientos de ejecución, como puede ocurrir al plantear estudios pre o cuasiexperimentales, los cuales, ciertamente, requieren de tiempo para la aplicación de programas, autorización de un comité de ética en investigación y otros aspectos como el dominio de diseños estadísticos. Por otro lado, el hecho de que menos del $10 \%$ de las tesis considere alguna prioridad de investigación en salud demuestra que los tesistas no están adecuadamente asesorados para elegir el tema de investigación, lo cual hace pensar que los asesores carecen de producción científica ${ }^{(19)}$, por lo que no son motivados a resolver los problemas prácticos de la sociedad.

Estos hallazgos influyen directamente sobre la investigación formativa en las universidades de Puno. Deficiencias como la falta de docentes de investigación, jurados y asesores de tesis con producción científica ${ }^{(20-22)}$ intervienen en la temática de los trabajos de grado que realizan los tesistas ${ }^{(23)}$. Por otro lado, existe la necesidad de mejorar la enseñanza de cursos como Metodología de la Investigación o Seminario de Tesis ${ }^{(24)}$; sin embargo, un aspecto que de alguna manera ha impulsado la investigación en los estudiantes es el trabajo de las sociedades científicas y otros grupos semilleros ${ }^{(25)}$.

La principal limitación de nuestra investigación fue el sesgo de la información, pues no se tienen datos acerca de lo que pudo influir en la elección de los temas en las tesis. Se recomienda investigar con mayor apoyo de las instituciones 
para determinar cuáles son los factores que influyen en la calidad de una investigación en pregrado.

Se concluye que la proporción de tesis de salud de universidades de Puno que abordan una prioridad de investigación en salud es moderada. Esto repercute directamente en la calidad de la investigación formativa, así como la prevalencia de problemas sanitarios en esta parte del Perú.

Contribuciones de los autores: OMB concibió la idea y el diseño de investigación y realizó el análisis estadístico. GRV, DRC, NCP, FQM y LCV recolectaron los datos e interpretaron los resultados. CRM se encargó de la revisión crítica y aprobó la versión final. Todos los autores revisaron la versión final y aprobaron el manuscrito.

Fuentes de financiamiento: Este artículo fue financiado por los autores.

Conflicto de intereses: Los autores declaran no tener ningún conflicto de interés.

\section{REFERENCIAS BIBLIOGRÁFICAS}

1. Mayta-Tristán P, Toro-Huamanchumo CJ, Alhuay-Quispe J, Pacheco-Mendoza J. Producción científica y licenciamiento de escuelas de medicina en el Perú. Rev Peru Med Exp Salud Pública. 2019;36(1):106.

2. Hernández R, Carranza R, Caycho-Rodriguez T, Cabrera-Orosco I, Arias D. Publicaciones científicas en revistas peruanas de psicología: un análisis desde la participación estudiantil. Rev Digit Investig en Docencia Univ. 2019;13(2):19-28.

3. Moquillaza-Alcántara VH. Producción científica asociada al gasto e inversión en investigación en universidades peruanas. An Fac Med. 2019;80(1):56-9.

4. Gonzales-Saldaña J, Chavez-Uceda T, Lemus-Arteaga K, Silva-Ocas I, Galvez-Olortegui T, Galvez-Olortegui J. Scientific production of the medical faculty of a Peruvian university in SCOPUS and PubMed. Educ Medica. 2018;19:128-34.

5. Luna-Solís Y. Producción científica en salud mental en Perú. Reto en tiempos de reforma de salud. Acta Méd Peruana. 2015;32(1):36-40.

6. Instituto Nacional de Salud. Prioridades de Investigación en Salud en el Perú 2010 - 2014: La experiencia de un proceso participativo y descentralizado/Sistematización de la experiencia [Internet]. INS: Lima; 2011. Disponible en: http://www.ins.gob.pe/insvirtual/ images/otrpubs/pdf/Prioridades \%5B13.6.11\%5D.pdf

7. Caballero P, Yagui M, Espinoza M, Castilla T, Granados A, Velásquez $A$, et al. Prioridades regionales y nacionales de Investigación en Salud, Perú 2010-2014: un proceso con enfoque participativo y descentralista. Rev Peru Med Exp Salud Pública. 2014;27(3):398-411.

8. Instituto Nacional de Salud. Definición de Prioridades Nacionales de Investigación en Salud 2015 - 2021 [Internet]. INS. Disponible en: https://web.ins.gob.pe/es/prensa/noticia/definicion-deprioridades-nacionales-de-investigacion-en-salud-2015-2021

9. Ministerio de Salud. Prioridades de Investigación en Salud 2019 2023 [Internet]. MINSA: Lima; 2019. Disponible en: https://web. ins.gob.pe/index.php/es/investigacion-en-salud/prioridades-deinvestigacion
10. Ato M, López J, Benavente A. Un sistema de clasificación de los diseños de investigación en psicología. An Psicol. 2013;29(3):1038-59.

11. Quispe-Juli CU, Aragón C, Moreno-Loaiza O. Escaso número de tesis enmarcadas en las prioridades de investigación en salud en una facultad de medicina peruana Scant. Revista Cubana de Información en Ciencias de la Salud (ACIMED). 2019;30(1):1-14.

12. Laguna-Torres VA, Francia-Romero J, Bisso-Andrade A, Guerreros A, Cerna-Barco J, Sanchez-Vergaray E, et al. Vigilancia, prevención y control del virus de la influenza en Perú. Rev Peru Med Exp Salud Pública. 2019;36(3):511-4.

13. Carrillo-Larco RM, Bernabé-Ortiz A. Diabetes mellitus tipo II en Perú: una revisión sistemática sobre la prevalencia e incidencia en población general. Rev Peru Med Exp Salud Pública. 2018;36(1):1-11.

14. Avila J, Tavera M, Carrasco M. Características epidemiológicas de la mortalidad neonatal en el Perú, 2011-2012. Rev Peru Med Exp Salud Pública. 2015;32(3):423-30.

15. Mamani Benito OJ. Calidad metodológica y características de las tesis de pregrado de psicología de una universidad privada del Perú. Propósitos y Represent. 2018;6(2):301.

16. Castro-Maldonado B, Callirgos-Lozada C, Caicedo-Pisfil M, PlasenciaDueñas A, Diaz-Velez C. Características de las tesis de pregrado de Medicina de una universidad pública del Perú. Horiz Med. 2015;15(3):34-9.

17. Mamani-Benito O, Ramos G, Rodrigo D, Quispe F, Chipana N. Publicación científica y características de las tesis de salud de universidades del departmento de Puno, Peru. Horiz Med. 2020;20(3):e1149.

18. Mamani-Benito OJ. La asesoría de tesis en pregrado: una labor que requiere un nuevo enfoque. Rev Medica Hered. 2019;30(2):124.

19. Mamani O. El asesor de tesis como Coach: una alternativa para impulsar la producción científica estudiantil. Educ Médica Super. 2018;33(1):e1590.

20. Mamani-Benito O, Ventura-León J, Caycho-Rodriguez T. Publicación cientifica de docentes que conforman el jurado de tesis en una Facultad de Ciencias de la Salud peruana. Revista Cubana de Información en Ciencias de la Salud (ACIMED). 2019;30(3):1-9.

21. Mamani OJ. Publicación científica y características de asesores de tesis de una Escuela De Psicología Peruana. Apunt Univ. 2019;9(2):27-36.

22. Pereyra-Elías R, Huaccho-Rojas J, Taype-Rondan Á, Mejia CR, Mayta-Tristán P. Publicación y factores asociados en docentes universitarios de investigación científica de escuelas de medicina del Perú. Rev Peru Med Exp Salud Pública. 2014;31(3):424-30.

23. Mejia CR, Cáceres OJ, Vera CA, Inga-Berrospi F, Mayta-Tristán P. Percepción y factores asociados a insatisfacción que los médicos recién graduados tienen de sus asesores de tesis, Lima-Perú. Rev Cuba Educ Médica Super. 2016;30(4):340-8.

24. Taype-Rondán A, Huaccho-Rojas J, Pereyra-Elias R, Mejia C, Mayta-Tristan P. Caracteristicas de los cursos de investigación en escuelas de medicina del Perú. Arch Med. 2015;11(2):1-7.

25. Mejia CR, Valladares-Garrido MJ, Almanza-Mio C, Benites-Gamboa D. Participación en una sociedad científica de estudiantes de Medicina asociada a la producción científica extracurricular en Latinoamérica. Educ Médica. 2018;20:99-103. 
Oscar Mamani-Benito; Glubert Ramos-Vilca; Dawer Rodrigo Ccaso; Neyda Chipana Paye; Fernando Quispe Mayta;

Laura Ccasa-Valero; Christian R. Mejia

\section{Correspondencia:}

Oscar Mamani Benito

Dirección: Carretera Arequipa km 6, Chullunquiani. Juliaca,

Perú.

Teléfono: (511) 959925651

Correo electrónico: oscar.mb@upeu.edu.pe

Recibido: 05 de octubre de 2020

Evaluado: 10 de noviembre de 2020

Aprobado: 02 de diciembre de 2020

( $)$ La revista. Publicado por Universidad de San Martín de Porres, Perú. (cc) Br Licencia de Creative Commons Artículo en acceso abierto bajo términos de Licencia Creative Commons Atribución 4.0 Internacional. (http://creativecommons.org/licenses/by/4.0/)

\section{ORCID iDs}

Oscar Mamani-Benito

Glubert Ramos-Vilca

Dawer Rodrigo Ccaso

Neyda Chipana Paye

Fernando Quispe Mayta

Laura Ccasa-Valero

Christian R. Mejia

\begin{abstract}
- https: / / orcid.org/0000-0002-9818-2601
- https: / / orcid.org/0000-0003-3190-6748

(1) https: / / orcid.org/0000-0002-2657-4171

(1) https: / / orcid.org/0000-0002-2119-8429

(1) https: / / orcid.org/0000-0002-3601-2199

(1) https: / /orcid.org/0000-0003-2599-4497

() https: / / orcid.org/0000-0002-5940-7281
\end{abstract}

